\title{
Attack on Six Rounds of CRYPTON
}

\author{
Carl D’Halluin, Gert Bijnens, Vincent Rijmen`, and Bart Preneel ${ }^{\star}$ \\ Katholieke Universiteit Leuven, ESAT-COSIC \\ K. Mercierlaan 94, B-3001 Heverlee, Belgium \\ carl.dhalluin@esat.kuleuven.ac.be \\ gert.bijnens@esat.kuleuven.ac.be \\ vincent.rijmen@esat.kuleuven.ac.be \\ bart.preneel@esat.kuleuven.ac.be
}

\begin{abstract}
In this paper we present an attack on a reduced round version of CRYPTON. The attack is based on the dedicated SQUARE attack. We explain why the attack also works on CRYPTON and prove that the entire 256-bit user key for 6 rounds of CRYPTON can be recovered with a complexity of $2^{56}$ encryptions, whereas for SQUARE $2^{72}$ encryptions are required to recover the 128-bit user key.
\end{abstract}

\section{Introduction}

The block cipher CRYPTON was recently proposed as a candidate algorithm for the AES [5. In this paper we describe a chosen plaintext attack that works if the cipher is reduced to 6 rounds instead of the specified 12 rounds. Our attack is based on the dedicated SQUARE attack presented in [2, but because of the differences between SQUARE and CRYPTON, the attack has to be modified in several points.

Previous analysis of CRYPTON led to the discovery of a failure of the key scheduling, resulting in a number of weak keys 16]. Our attack works on a reduced version of CRYPTON for all keys. For a final optimisation of the attack, we exploit another feature of the key scheduling.

In Section 2 we give a short description of CRYPTON. We present the basic attack in Section 3. Section 4 discusses the recovery of the user key. Section 5 and Section 6 discuss the extension of the attack to five rounds, and Section 7 gives the six round attack. We conclude in Section 8 ,

\section{Description of CRYPTON}

The block cipher CRYPTON is based on SquARE 2. The plaintext data is ordered in 16 bytes, which are put in a square scheme, called the state. If $A$ is the state at a certain moment, the different bytes of $A$ are called $(A)_{i j}$ with $i$ and $j$ varying from 0 to 3 (see Figure 1). CRYPTON uses 6 elementary transformations.

\footnotetext{
* F.W.O. Postdoctoral Researcher, sponsored by the Fund for Scientific Research -
} Flanders (Belgium) 


\begin{tabular}{|l|l|l|l|}
\hline$A_{03}$ & $A_{02}$ & $A_{01}$ & $A_{00}$ \\
\hline$A_{13}$ & $A_{12}$ & $A_{11}$ & $A_{10}$ \\
\hline$A_{23}$ & $A_{22}$ & $A_{21}$ & $A_{20}$ \\
\hline$A_{33}$ & $A_{32}$ & $A_{31}$ & $A_{30}$ \\
\hline
\end{tabular}

Fig. 1. Byte coordinates of the state

$-\sigma_{K_{e}^{i}}$ is a key addition (EXOR) with round key $i$. This operation is the same as the key addition in SQUARE.

$-\pi_{e}$ and $\pi_{o}$ are linear transformations that act on columns of the state. The $\pi$-transformations operate on two bits at a time, calculating a new value by exoring the old values of two bits in corresponding positions in three different bytes of the column. These operations are the replacement of the MDSbased $\theta$ in SquARE. They can be implemented using four masks, denoted $M_{0}, \ldots M_{3}$.

- $\gamma_{e}$ and $\gamma_{o}$ are non-linear transformations that apply S-boxes to the different state bytes. They have the additional property that $\gamma_{e}=\gamma_{o}^{-1}$. These operations correspond to the single $\gamma$ in SQUARE.

$-\tau$ is a simple transposition (upper row becomes rightmost column, lower row becomes leftmost column, ...). This operation is the same as the $\pi$ of Square. Note that $(D, C, B, A)^{t}$ means that $A^{t}$ is the upper row and $D^{t}$ is the lower row.

Throughout this text we use versions of CRYPTON with less rounds than the standard number of rounds which is 12 . The standard version of CRYPTON is :

$$
\begin{gathered}
\text { Encrypt }=\phi_{e} \circ \rho_{e K_{e}^{12}} \circ \rho_{o K_{e}^{11}} \circ \cdots \circ \rho_{e K_{e}^{2}} \circ \rho_{o K_{e}^{1}} \circ \sigma_{K_{e}^{0}} \\
\operatorname{with}\left\{\begin{array}{c}
\phi_{e}=\tau \circ \pi_{e} \circ \tau \\
\rho_{e K}=\sigma_{K} \circ \tau \circ \pi_{e} \circ \gamma_{e} \\
\rho_{o K}=\sigma_{K} \circ \tau \circ \pi_{o} \circ \gamma_{o}
\end{array}\right.
\end{gathered}
$$

Since $\phi_{e}$ uses no key material, it is easily invertible by a cryptanalyst and therefore we do not consider it in the following text. For example a five round version of CRYPTON means in this text :

$$
\text { Encrypt }_{5}=\rho_{o K_{e}^{5}} \circ \rho_{e K_{e}^{4}} \circ \rho_{o K_{e}^{3}} \circ \rho_{e K_{e}^{2}} \circ \rho_{o K_{e}^{1}} \circ \sigma_{K_{e}^{0}} \text {. }
$$

Unless stated otherwise the output state of round $n$ is denoted $R_{n}$ in this paper. $R_{0}$ represents the output after the initial key addition $\sigma_{K_{e}^{0}}$. PT represents the plaintext and $C T$ represents the ciphertext. So $C T=\phi_{e}\left(R_{12}\right)$. 


\section{Basic Attack: 4 Rounds of CRYPTON}

In this section we will explain how the dedicated SQUARE attack 2] can be modified to attack CRYPTON. Due to the differences between both algorithms, the attack works in a slightly different way. The final 6-round attack allows to recover the entire 256-bit user key immediately, using less computer time than the equivalent attack on SQUARE, which only recovers a 128-bit key. This makes the attack much more significant on CRYPTON than on SQUARE.

First we explain the attack on 4 rounds of CRYPTON and the reason why it works. The attack on 4 rounds uses approximately $2^{9}$ chosen plaintexts and their corresponding ciphertexts. We show a way to recover a 128-bit user key without using additional plaintexts. In the next Sections a 5-round and a 6-round attack on CRYPTON are described, which require significantly more chosen plaintexts.

Let a $\Lambda$-set be a set of 256 states that are all different in some of the (16) state bytes (the active) and all equal in the other state bytes (the passive). The 256 elements of the $\Lambda$-set are denoted $\Lambda_{a}$ with $a$ varying from 0 to 255 . Let $\lambda$ be the set of indices of the active bytes (indices varying from 0 to 3 ). We have:

$$
\forall a, b \in\{0 \ldots 255\}: \quad\left\{\begin{array}{l}
\left(\Lambda_{a}\right)_{i j} \neq\left(\Lambda_{b}\right)_{i j} \text { for }(i, j) \in \lambda \\
\left(\Lambda_{a}\right)_{i j}=\left(\Lambda_{b}\right)_{i j} \text { for }(i, j) \notin \lambda .
\end{array}\right.
$$

We start with a $\Lambda$-set with a single active byte. From the definition it follows that this byte will take all 256 possible values $\{0 \times 00,0 x 01, \ldots, 0 x f\}$ over the different states in the $\Lambda$-set. As a consequence, the $\Lambda$-set is balanced, by which we mean:

$$
\bigoplus_{a=0}^{255}\left(\Lambda_{a}\right)_{i j}=0 \times 00, \quad \forall i, j .
$$

This is valid for the one active byte because $\bigoplus_{a=0}^{255} a=0 \times 00$ and for the fifteen passive bytes because $\bigoplus_{a=0}^{255} b=0 \mathrm{x} 00$ if $b$ is a constant byte.

We now investigate the balancedness, the positions and the properties of the active bytes through the subsequent transformations. After each transformation we have a new set of active bytes, which is called the state scheme. If we say that the state scheme is balanced we mean that the transformation of the original $\Lambda$-set up to this point is still balanced. It is also important in the state scheme to know some properties of each byte e.g. this byte is a constant for each element of the $\Lambda$-set, or that byte takes every value of the set $\{0 \mathrm{x} 00,0 \mathrm{x} 01, \ldots, 0 \mathrm{xff}\}$ over the $\Lambda$-set (mathematically : $\left.\bigcup_{a=0}^{255}\left(\Lambda_{a}\right)_{i j}=\{0 \mathrm{x} 00,0 \mathrm{x} 01, \ldots, 0 \mathrm{xff}\}\right)$.

Round 0 (initial key addition) The state scheme evolution through round 0 is displayed in Figure 2, We have a single active byte which takes 256 different values over the $\Lambda$-set (full black square in the figure). The 15 other bytes are passive and therefore they have a constant value over the $\Lambda$-set.

The initial key addition $\sigma_{K_{e}^{0}}$ does not change the balancedness of the state scheme because the EXOR-sum of 256 times the same key byte cancels out. 


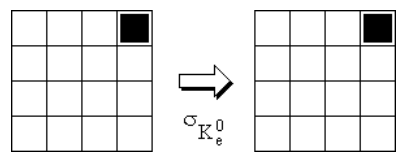

Fig. 2. State scheme through round 0

$\sigma_{K_{e}^{0}}$ cannot change the state scheme either because the EXOR-addition with a constant byte acts as a simple bijection in the set of all possible byte values $\{0 \mathrm{x} 00,0 \mathrm{x} 01, \ldots, 0 \mathrm{xff}\}$. Hence, if we have 256 different byte values, the key addition will map them on 256 different byte values, and if we have 256 times the same byte value, the key addition will obviously map them on 256 times the same byte value.

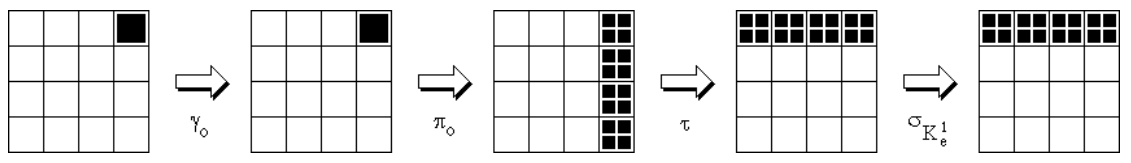

Fig. 3. State scheme through round 1

Round $1 \gamma_{o}$ does not change the state scheme nor the balancedness of the scheme since $\gamma_{o}$ is bijective for each of the bytes. So 256 different byte values are mapped onto the same 256 different byte values. After $\pi_{o}$ we have one active column. The other three columns are still passive. Now we investigate the properties of this active column. Therefore we have to take a closer look at the linear transformation $\pi_{o}$ which acts separately on the four columns. Let $A$ denote the input state of $\pi_{o}$ and let $B$ denote the output state. Now we can write $\pi_{o}$ as

$$
\begin{aligned}
B & =\pi_{o}(A) \\
& \mathbb{} \\
(B)_{i j} & =\bigoplus_{k=0}^{3}\left((A)_{k j} \wedge M_{(i+k)} \bmod _{4}\right),
\end{aligned}
$$

where $\wedge$ is the binary AND-operator and $M_{i}$ with $i=0,1, \ldots, 3$ is a masking word, leaving 6 bits of every byte unmasked. For example $M_{0}=0 x 3 f \mathrm{cff} 3 \mathrm{fc}$. In state $A$ we have one active byte which takes 256 different values over the $\Lambda$-set. Now $\pi_{o}$ acts on this $\Lambda$-set generating one active column, in which each of the four bytes contains 6 bits of the original active byte of state $A$. Hence each byte of the active column of state $B$ must take 64 different values, each occurring exactly 4 times.

Taking the EXOR of 4 times the same byte results in 0x00; state $B=\pi_{o}(A)$ is still balanced. In Figure 3 the new active bytes, which take 64 different values 
each occurring 4 times, are displayed with a white + symbol in a black square. $\tau$ is a simple matrix transposition, which only changes the positions of the bytes, but does not change their value. Thus, $\tau$ does not change the balancedness or the properties of the state scheme. $\sigma_{K_{e}^{1}}$ does not change the balancedness or the properties either.
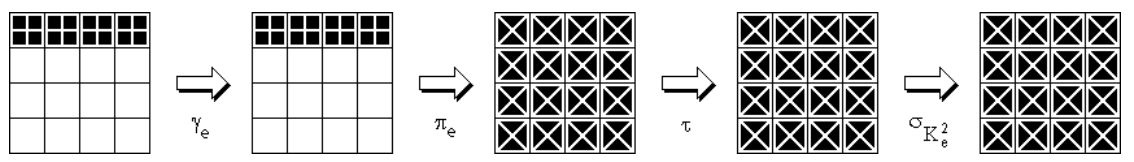

Fig. 4. State scheme through round 2

Round 2 Figure 4 shows the state scheme evolution through round 2. $\gamma_{e}$ does not change the state scheme since $\gamma_{e}$ will map a byte value occurring 4 times onto another byte value occurring 4 times. This does not change the EXOR-sum of the bytes, which remains $0 \times 00$.

$\pi_{e}$ generates 16 active bytes (all bytes of the state are now active). Due to the specific structure of $\pi_{e}$ (similar to that of $\pi_{o}$ ), each byte of the output state of $\pi_{e}$ takes $n \leq 64$ different values. On a fixed position in the output state of $\pi_{e}$, every possible byte value (from $0 \times 00$ to $0 x f f$ ) occurs either zero or a multiple of 4 times, due to $\pi_{e}$. On Figure 4 these bytes are shown as a white $\times$ symbol on a black square. This occurrence in multiples of 4 can be explained with the following example.

Suppose that we are working with nibbles 1 . We have a $\Lambda$-set consisting of 16 nibbles e.g. $\Lambda=\{1110,1110,1110,1110,1010,1010,1010,1010,1111,1111$, 1111, 1111, 0001, 0001, 0001, 0001\}. We now have a similar situation as the real input $\Lambda$-set of $\pi_{e}$. Now we look at the effect of a binary nibble mask e.g. $M=1110$ which leaves 3 of the 4 bits unmasked.

\begin{tabular}{|l||l|}
\hline $1110 \wedge 1110=1110$ & $1111 \wedge 1110=1110$ \\
$1110 \wedge 1110=1110$ & $1111 \wedge 1110=1110$ \\
$1110 \wedge 1110=1110$ & $1111 \wedge 1110=1110$ \\
$1110 \wedge 1110=1110$ & $1111 \wedge 1110=1110$ \\
$1010 \wedge 1110=1010$ & $0001 \wedge 1110=0000$ \\
$1010 \wedge 1110=1010$ & $0001 \wedge 1110=0000$ \\
$1010 \wedge 1110=1010$ & $0001 \wedge 1110=0000$ \\
$1010 \wedge 1110=1010$ & $0001 \wedge 1110=0000$ \\
\hline
\end{tabular}

The table shows the effect of the bit masking. After the masking $\Lambda=\{1110$, 1110, 1110, 1110, 1010, 1010, 1010, 1010, 1110, 1110, 1110, 1110, 0000, 0000, $0000,0000\}$. Now the value 1110 occurs 8 times, the values 1010 and 0000 both

\footnotetext{
${ }^{1}$ nibble $=$ four-bit quantity
} 
occur 4 times, and the other possible nibble values do not occur at all. We see that the values in the resulting $\Lambda$-set occur in multiples of 4 .

If we look at the real $\pi_{e}$ then the only thing we do is masking (leaving unmasked 6 bits of every byte) and EXOR-additions. We can generalize our conclusion from above and say that on every byte position every byte value in the output state of $\pi_{e}$ occurs in multiples of 4 over the $\Lambda$-set. This leaves the state balanced. $\tau$ and $\sigma_{K_{e}^{2}}$ do not change the state scheme or the balancedness for reasons mentioned before.

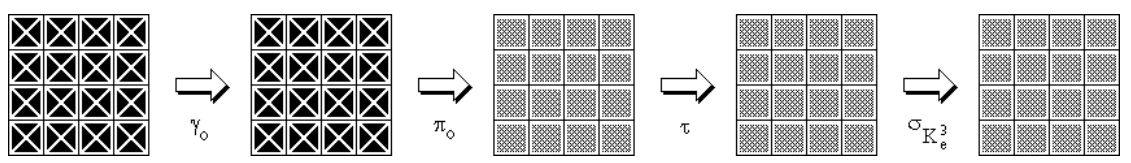

Fig. 5. State scheme through round 3

Round $3 \gamma_{o}$ does not change the state scheme properties or the balancedness since $\gamma_{o}$ will map a byte value occurring a multiple of 4 times on another byte value occurring the same multiple of 4 times. This does not change the EXORsum of the bytes, which remains $0 \times 00$.

After $\pi_{o}$ all bytes are still active but the different values no longer occur in multiples of four. This destroys our structure and limits the power of the whole attack. These active bytes are displayed in Figure 5 as grey squares. Nevertheless the scheme is still balanced because of the linearity of $\pi_{o}$. This can be proven as follows. Let $A_{a}$ denote the balanced input state of $\pi_{o}$ for the $a$-th element of the $\Lambda$-set, and let $B_{a}$ denote the corresponding output state. Then we have the following:

$$
\begin{aligned}
& B_{a}=\pi_{o}\left(A_{a}\right) \\
& \Downarrow \\
& \bigoplus_{a=0}^{255}\left(\left(B_{a}\right)_{i j}\right)=\bigoplus_{a=0}^{255} \bigoplus_{k=0}^{3}\left(\left(\left(A_{a}\right)_{k j}\right) \wedge M_{(i+k) \bmod 4}\right) \\
& =\bigoplus_{k=0}^{3}(\underbrace{\bigoplus_{a=0}^{255}\left(\left(A_{a}\right)_{k j}\right)}_{=0 \mathrm{x} 00}) \wedge M_{(i+k) \bmod 4} \\
& =0 \times 00 \text {. }
\end{aligned}
$$

$\tau$ and $\sigma_{K_{e}^{3}}$ do not change the state scheme properties or the balancedness. 
Round 4 The output of round 3 is balanced:

$$
\bigoplus_{a=0}^{255}\left(R_{3}\right)_{a}=(0)
$$

with $(0)$ the all-zero state scheme $\left((0)_{i j}=0 \mathrm{x} 00, \forall i, j\right)$. We drop the index $a$ because the following formulae are valid for each element of the $\Lambda$-set. We have for the output of round 4:

$$
\begin{aligned}
R_{4} & =\rho_{e K_{e}^{4}}\left(R_{3}\right) \\
& =\sigma_{K_{e}^{4}} \circ \tau \circ \pi_{e} \circ \gamma_{e}\left(R_{3}\right) .
\end{aligned}
$$

Taking the inverse of this formula and using the linearity of $\pi_{e}$ and $\tau$ leads us to

$$
R_{3}=\gamma_{o}\left(\pi_{e}\left(\tau\left(R_{4}\right)\right) \oplus \pi_{e}\left(\tau\left(K_{e}^{4}\right)\right)\right)
$$

Since $R_{4}$ is known, we can determine $\pi_{e}\left(\tau\left(R_{4}\right)\right)$ completely. Now we guess one of the 16 bytes of $\pi_{e}\left(\tau\left(K_{e}^{4}\right)\right)$. Using (1) we can calculate $R_{3}$ for this byte position for all 256 members of our $\Lambda$-set. If the EXOR of all these values is zero then we have found a possible value for this key byte. We can do this independently for all 16 key bytes. Experiments show that only one or two values are possible for each key byte. If we do the same again starting with a different $\Lambda$-set, we can find the 128 bits of round key $K_{e}^{4}$ with overwhelming probability.

\section{Calculation of the User Key of 128 Bits}

In this section we show how to calculate the user key of 128 bits when we know one round key of 128 bits. In the previous section we have extracted round key $K_{e}^{4}$. The user key can now be calculated by taking into account the key expansion:

$$
\begin{aligned}
\left(V_{e}[3], V_{e}[2], V_{e}[1], V_{e}[0]\right)^{t} & =\left(\tau \circ \gamma_{o} \circ \sigma_{P} \circ \pi_{o}\right)\left((U[6], U[4], U[2], U[0])^{t}\right) \\
\left(V_{e}[7], V_{e}[6], V_{e}[5], V_{e}[4]\right)^{t} & =\left(\tau \circ \gamma_{e} \circ \sigma_{Q} \circ \pi_{e}\right)\left((U[7], U[5], U[3], U[1])^{t}\right) \\
T_{0} & =V_{e}[0] \oplus V_{e}[1] \oplus V_{e}[2] \oplus V_{e}[3] \\
T_{1} & =V_{e}[4] \oplus V_{e}[5] \oplus V_{e}[6] \oplus V_{e}[7] \\
E_{e}[i] & =V_{e}[i] \oplus T_{1} \text { for } i=0,1,2,3 \\
E_{e}[i] & =V_{e}[i] \oplus T_{0} \text { for } i=4,5,6,7
\end{aligned}
$$

with $U[i], i=0,1, \ldots, 7$ the user key and $E_{e}[i]$ the expanded keys. In [3] it is stated that if the user key is shorter than 256 bits it must be prepend by zero-words e.g. a 64-bit user key means $U[i]=0 \mathrm{x} 00000000$ for $i>1$.

We now try to calculate a 128-bit user key given $K_{e}^{4}$. Using appropriate shifts and constant additions we can easily find $E_{e}[0], E_{e}[1], E_{e}[2]$ and $E_{e}[3]$ given $K_{e}^{4}$ 
with the following formulae (see appendices in [3]):

$$
\begin{aligned}
K_{e}^{4} & =\left(K_{e}[19], K_{e}[18], K_{e}[17], K_{e}[16]\right)^{t} \\
K_{e}[16] & =E_{e}[0] \ll 8 \oplus \oplus C_{1} \\
K_{e}[17] & =E_{e}[1] \ll 24 \oplus R C_{0} \\
K_{e}[18] & =E_{e}[2] \ll 16 \oplus R C_{1} \\
K_{e}[19] & =E_{e}[3]^{\ll 8} \oplus R C_{0} .
\end{aligned}
$$

Here $A^{\ll i}$ denotes the left-wise bit rotation of a 32-bit word $A$ over $i$ positions. The problem to be solved can be stated as follows: Given $E_{e}[i]$ for $0 \leq i \leq 3$ calculate $U[i]$ for $0 \leq i \leq 3$ knowing that $U[j]$ for $4 \leq j \leq 7$ are all zero. We are able to solve this problem with a byte-wise reconstruction of the unknown values $T_{0}$ and $T_{1}$.

Rightmost byte of $\boldsymbol{T}_{\mathbf{1}}$ (byte 0 ) First of all we have to guess byte 0 of $T_{1}$. This enables us to calculate byte 0 of $V_{e}[i]$ for $i=0,1, \ldots, 3$. Since

$$
\left(V_{e}[3], V_{e}[2], V_{e}[1], V_{e}[0]\right)^{t}=\left(\tau \circ \gamma_{o} \circ \sigma_{P} \circ \pi_{o}\right)\left((U[6], U[4], U[2], U[0])^{t}\right),
$$

we find that

$$
\sigma_{P} \circ \gamma_{e} \circ \tau\left(V_{e}[3], V_{e}[2], V_{e}[1], V_{e}[0]\right)^{t}=\pi_{o}\left((U[6], U[4], U[2], U[0])^{t}\right)
$$

We know the upper row of the left side of this expression. From the right side we know something about the structure of $\pi_{o}\left((U[6], U[4], U[2], U[0])^{t}\right)$ since $U[i]=$ $0 \mathrm{x} 0$ for $i=4,5, \ldots, 7$. This structure is:

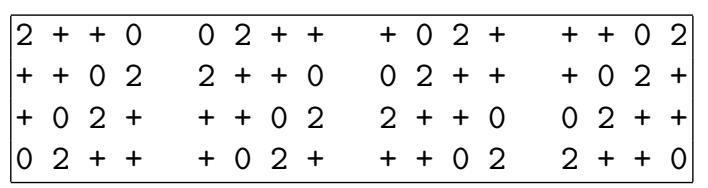

The symbols in this scheme denote 2-bit quantities in the total state. The four symbols in the same row of a sub-group form together one byte of the state. E.g., $2++0$ in the top left corner denotes the leftmost byte of $U[0]^{t}$. A 0 or a 2 respectively indicate to copy the corresponding two bits of $U[0]$ or $U[2]$. A + indicates to write the EXOR of the corresponding two bits of $U[0]$ and $U[2]$. The scheme can be derived by taking into account the different masks used in the linear transformation $\pi_{o}$ (see [3]).

Byte 1 of $\boldsymbol{T}_{\mathbf{1}}$ This byte can by found by checking the second row of $\pi_{o}\left((U[6], U[4], U[2], U[0])^{t}\right)$. The four 2-bit-positions in the scheme where we have a + symbol in the upper and the second row must contain the same 2-bit values. This results in approximately one possible value for byte 1 of $T_{1}$. 
Byte 2 of $\boldsymbol{T}_{\mathbf{1}}$ This byte can be found by checking the third row of $\pi_{o}\left((U[6], U[4], U[2], U[0])^{t}\right)$. We can calculate in advance 12 2-bit-positions of the third row of the scheme since $s_{1} \oplus s_{2}=s_{3}$ with $s_{1} s_{2} s_{3}$ a random permutation of the symbols +02 . This also results in approximately one possible value for byte 2 of $T_{1}$.

Leftmost byte of $\boldsymbol{T}_{\mathbf{1}}$ (byte 3 ) Since we have the upper three rows of the scheme, we can calculate the lower row (using the same formula $s_{1} \oplus s_{2}=s_{3}$ ), and calculate back to the leftmost column of $\left(V_{e}[3], V_{e}[2], V_{e}[1], V_{e}[0]\right)^{t}$. If we find four times the same value for the leftmost byte of $T_{1}$ by checking the $E_{e}[i]$ values, we have a possible user key. We do not expect that more than one valid user key can be found.

\section{Addition of a Fifth Round at the End}

In this section we add a fifth round in the end to the basic attack by guessing one column of round key $K_{e}^{5}$ at once. To recover $K_{e}^{4}$ we have to know only one of the 16 bytes of $\pi_{e}\left(\tau\left(R_{4}\right)\right)$ at a time, so knowledge of one row of $R_{4}$ is sufficient. To add a fifth round to the attack we use the following formula:

$$
\begin{aligned}
& R_{5}=\sigma_{K_{e}^{5}} \circ \tau \circ \pi_{o} \circ \gamma_{o}\left(R_{4}\right) \\
& \quad \Downarrow \\
& R_{4}=\gamma_{e}\left(\pi_{o}\left(\tau\left(R_{5}\right)\right) \oplus \pi_{o}\left(\tau\left(K_{e}^{5}\right)\right)\right),
\end{aligned}
$$

which is valid because of the linearity of $\pi_{o}$ and $\tau$.

If we guess a row of $\pi_{o}\left(\tau\left(K_{e}^{5}\right)\right)$ we can calculate a single row of $R_{4}$ and a single column of $\pi_{e}\left(\tau\left(R_{4}\right)\right)$. Since $R_{3}=\gamma_{o}\left(\pi_{e}\left(\tau\left(R_{4}\right)\right) \oplus \pi_{e}\left(\tau\left(K_{e}^{4}\right)\right)\right)$ must be balanced, we can exclude approximately $\frac{255}{256}$ of our $2^{40}$ guessed key values $\left(2^{32}\right.$ for the row of $\pi_{o}\left(\tau\left(K_{e}^{5}\right)\right)$ and $2^{8}$ for the one byte of $\pi_{e}\left(\tau\left(K_{e}^{4}\right)\right)$ gives $2^{40}$ guessed key values). This means that we have to repeat this procedure for at least $5 \Lambda$-sets in order to find the round keys $K_{e}^{5}$ and $K_{e}^{4}$ from which we can calculate the entire 256-bit user key due to the simple key scheduling mechanism (see appendices in [3]).

\section{Addition of a Fifth Round in the Beginning}

In this section we add a fifth round in the beginning to the basic attack. We try to generate $\Lambda$-sets with only one active byte (taking 256 different values) at the output of round 1 . We start with a pool of $2^{32}$ plaintexts that differ only in the byte values of the first column. We assume a value for the 4 bytes of the first column of the first roundkey. This enables us to compose a few sets.

Let $A$ be the desired output state of $\pi_{o}$ of the first round and let $P T$ be the plaintext state.

$$
A=\left(\pi_{o} \circ \gamma_{o} \circ \sigma_{K_{e}^{0}}\right)(P T)
$$




$$
\begin{aligned}
& \text { » } \\
& P T=\left(\sigma_{K_{e}^{0}} \circ \gamma_{e} \circ \pi_{o}\right)(A) \\
& =K_{e}^{0} \oplus \gamma_{e}\left(\pi_{o}(A)\right)
\end{aligned}
$$

Since in $\gamma_{e}\left(\pi_{o}(A)\right)$ only the first column is active, we can reuse the texts of our pool for every value of $K_{e}^{0}$. Given a $\Lambda$-set, we can recover the value of $K_{e}^{5}$ with our four round attack on rounds $2,3,4$ and 5 . We repeat the attack several times with different $\Lambda$-sets. If the values suggested for $K_{e}^{5}$ are inconsistent, we have made a wrong assumption for the column of $K_{e}^{0}$. With this method we can find $K_{e}^{0}$ and $K_{e}^{5}$, hence we can find the full 256 bits of the user key.

\section{6-Round Version of CRYPTON}

The six round attack is a combination of the two previous extensions of the basic 4 -round attack. Due to the specific generation of round key $K_{e}^{6}$ we can make an improvement of $2^{16}$ on the dedicated SQUARE attack, and recover the full 256 key bits.

We first guess 1 byte column of $K_{e}^{0}\left(2^{32}\right.$ possibilities). For each guess we can generate some $\Lambda$-sets at the output of $\pi_{o}$ of round 1 with the formula:

$$
P T=\gamma_{e}\left(\pi_{o}(A)\right) \oplus K_{e}^{0}
$$

Addition of a round at the end requires the knowledge of a row of $\pi_{e}\left(\tau\left(K_{e}^{6}\right)\right)$. If we know a column of $K_{e}^{0}$ then we also know 4 bytes of $K_{e}^{6}$ :

$$
\begin{aligned}
K_{e}^{0} & =\left(K_{e}[3], K_{e}[2], K_{e}[1], K_{e}[0]\right)^{t} \\
K_{e}[0] & =E_{e}[0] \\
K_{e}[1] & =E_{e}[1] \\
K_{e}[2] & =E_{e}[2] \\
K_{e}[3] & =E_{e}[3] \\
K_{e}^{6} & =\left(K_{e}[27], K_{e}[26], K_{e}[25], K_{e}[24]\right)^{t} \\
K_{e}[24] & =E_{e}[0] \ll 24 \oplus R C_{1} \\
K_{e}[25] & =E_{e}[1] \ll 24 \oplus R C_{02} \\
K_{e}[26] & =E_{e}[2]^{\ll 8} \oplus R C_{1} \\
K_{e}[27] & =E_{e}[3]^{\ll 8} \oplus R C_{02}
\end{aligned}
$$

If we want to know a row of $\pi_{e}\left(\tau\left(K_{e}^{6}\right)\right)$ we have to know a column of $K_{e}^{6}$ and we have to guess only 16 bits instead of the full 32 bits as in the SQUARE attack if we choose the right columns of $K_{e}^{6}$.

This 6 -round attack will recover $K_{e}^{0}$ and the equivalent $K_{e}^{6}$, but also $K_{e}^{5}$. From these values we can calculate $E_{e}[i]$ for $i=0,1, \ldots, 7$, hence we can calculate the entire 256-bit user key. 


\section{Conclusion}

We have described attacks on several reduced round versions of the block cipher Crypton. Table 1 summarizes the requirements of the attacks. The 5-round (a) attack is described in section 5 and the 5-round (b) attack in section 6.

In its present form the described attack means no real threat to the full 12-round version of CRYPTON. However, after the discovery of weak keys [16 of CRYPTON, this is the second time that the key scheduling of CRYPTON is brought into discredit.

Table 1. Requirements for the described attacks on CRYPTON.

\begin{tabular}{|cccc|}
\hline Attack & \# Plaintexts & Time & Memory \\
\hline 4-round & $2^{9}$ & $2^{9}$ & small \\
5-round (a) & $2^{11}$ & $2^{40}$ & small \\
5-round (b) & $2^{32}$ & $2^{40}$ & $2^{32}$ \\
6-round & $2^{32}$ & $2^{56}$ & $2^{32}$ \\
\hline
\end{tabular}

\section{References}

1. J. Borst, "Weak keys of Crypton," technical comment submitted to NIST.

2. J. Daemen, L. Knudsen and V. Rijmen, "The block cipher Square," Fast Software Encryption, LNCS 1267, E. Biham, Ed., Springer-Verlag, 1997, pp. 149-165.

3. Lim, "CRYPTON : A New 128-bit Block Cipher," available from [5].

4. Lim, "Specification and Analysis of Crypton Version 1.0," FSE '99, these proceedings.

5. NIST's AES home page, http://www.nist.gov/aes .

6. S. Vaudenay, "Weak keys in Crypton," announcement on NIST's electronic AES forum, cf. [5].

\section{A Attack on Six Rounds of CRYPTON Version 1.0}

In 44 a new version of CRYPTON is proposed, CRYPTON version 1.0. We explain briefly how to extend our results to version 1.0, which features two major changes.

1. The nonlinear transformations $\gamma_{o}$ and $\gamma_{e}$ use now two S-boxes instead of only one. This doesn't influence our attack

2. The key scheduling has been changed, both in the generation of the expanded keys and in the generation of the roundkeys from the expanded keys. This influences our attack, but we will see that the attack still applies. 


\section{A.1 Round Key Derivation in Version 1.0}

The relation between roundkey 0 and roundkey 6 is very important for the complexity of our attack. In the new version this relation is more complex and uses a new operation $A^{\ll_{b} i}$, which is defined as a left-wise bit rotation of each of the four bytes of the 32-bit word $A$. The new calculation of roundkey 0 and roundkey 6 is:

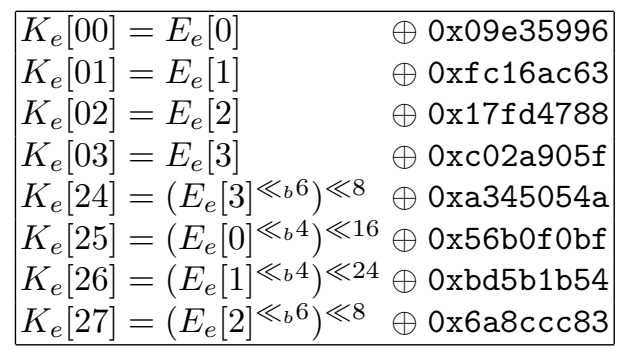

Notice that if we know one column of $\left(K_{e}[03], K_{e}[02], K_{e}[01], K_{e}[00]\right)^{t}$ then we know 16 bytes of a certain column of $\left(K_{e}[27], K_{e}[26], K_{e}[25], K_{e}[24]\right)^{t}$ because of the double occurrence of the ${ }^{\ll 8}$ operator in the previous table. This is the reason why our six-round attack still works on version 1.0 with the gain of $2^{16}$ time.

\section{B Calculation of the User Key of 128 Bits}

\section{B.1 Generation of the Expanded Key}

We show in this section how we can calculate the 128-bit user key when we know one roundkey of 128 bits. In the specifications of CRYPTON version 1.0 [4 the new generation of the expanded keys is as follows.

Let $K=k_{u-1} \ldots k_{1} k_{0}$ be a user key of $u$ bytes $(u=0,1, \ldots, 32)$. We assume that $K$ is 256 bits long (by prepending by as many zeros as required).

1. Split the user key into $U$ and $V$ as: for $i=0,1,2,3$,

$$
U[i]=k_{8 i+6} k_{8 i+4} k_{8 i+2} k_{8 i}, \quad V[i]=k_{8 i+7} k_{8 i+5} k_{8 i+3} k_{8 i+1} .
$$

2. Transform $U$ and $V$ using round transformations $\rho_{o}$ and $\rho_{e}$, respectively, with all-zero round keys :

$$
U^{\prime}=\rho_{o}(U), \quad V^{\prime}=\rho_{e}(V) .
$$

3. Compute 8 expanded keys $E_{e}[i]$ for encryption as: for $i=0,1,2,3$,

$$
E_{e}[i]=U^{\prime}[i] \oplus T_{1}, \quad E_{e}[i+4]=V^{\prime}[i] \oplus T_{0},
$$

where $T_{0}=\bigoplus_{i=0}^{3} U^{\prime}[i]$ and $T_{1}=\bigoplus_{i=0}^{3} V^{\prime}[i]$.

Since we know that $U[i]$ and $V[i]$ are all-zero for $i=2,3$ we know the lower 2 rows of $U$ and $V$. Since $U^{\prime}=\tau \circ \pi_{o} \circ \gamma_{o}(U)$ we can calculate $T_{1}, T_{0}$ and the user key by a byte-wise reconstruction of $T_{1}$. 


\section{B.2 Reconstruction of the 128-Bit User Key}

We have $\pi_{o} \circ \gamma_{o}(U)=\tau\left(U^{\prime}\right)$ with $U=(0 \times 0,0 \times 0, U[1], U[0])^{t}$. Let $\gamma_{o}(U)=$ $\left(\mathrm{b}, \mathrm{a}, 1^{\prime}, 0^{\prime}\right)^{t}$ with $\mathrm{b}=0 \times 0 \mathrm{~d} 63 \mathrm{~b} 1 \mathrm{~b} 1$ and $\mathrm{a}=0 \times \mathrm{xb} 18 \mathrm{~d} 63 \mathrm{~b} 1$. The $\mathrm{a}$ and $\mathrm{b}$ values can be calculated from the definition of $\gamma_{o}$ and from the S-boxes [4]. Now we try to find the unknown values 0 ' and $1^{\prime}$.

If we guess byte 0 of $T_{1}$ (rightmost byte of $T_{1}$ ) it is possible to calculate the upper row of $\pi_{o} \circ \gamma_{o}(U)$. The structure of this state is:

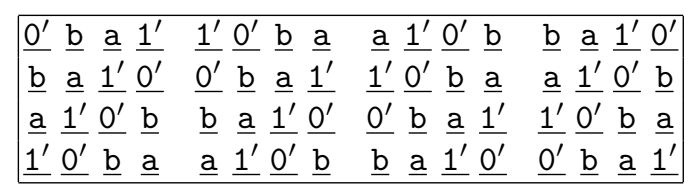

The rows in this scheme are counted from top to bottom starting with row 0 . The symbols in the scheme denote to copy the corresponding 2-bit values of the following 32-bit values:

$$
\begin{aligned}
& \underline{0^{\prime}}=\left(0^{\prime} \oplus 1^{\prime} \oplus \mathrm{a} \oplus \mathrm{b}\right) \oplus 0^{\prime}, \\
& \underline{1^{\prime}}=\left(0^{\prime} \oplus 1^{\prime} \oplus \mathrm{a} \oplus \mathrm{b}\right) \oplus 1^{\prime}, \\
& \underline{\mathrm{a}}=\left(0^{\prime} \oplus 1^{\prime} \oplus \mathrm{a} \oplus \mathrm{b}\right) \oplus \mathrm{a}, \\
& \underline{\mathrm{b}}=\left(0^{\prime} \oplus 1^{\prime} \oplus \mathrm{a} \oplus \mathrm{b}\right) \oplus \mathrm{b} .
\end{aligned}
$$

Since we know the upper row of the scheme (due to our initial guess of byte 0 of $T_{1}$ ) we can calculate byte 1 of $T_{1}$ because we can calculate 4 times 2 bits of $T_{1}$ on the positions of the second row of the scheme where we find a a symbol (the symbols 1 in figure [6), because:

$$
\begin{aligned}
\underline{\mathrm{a}} & =\left(0^{\prime} \oplus 1^{\prime} \oplus \mathrm{a} \oplus \mathrm{b}\right) \oplus \mathrm{a} \\
& =\left(0^{\prime} \oplus 1^{\prime} \oplus \mathrm{a} \oplus \mathrm{b}\right) \oplus \mathrm{b} \oplus \mathrm{b} \oplus \mathrm{a} \\
& =\underline{\mathrm{b}} \oplus(\mathrm{a} \oplus \mathrm{b}),
\end{aligned}
$$

and we know $\mathrm{a} \oplus \mathrm{b}$. Now we can calculate row 1 of our scheme $\pi_{o} \circ \gamma_{o}(U)$ completely.

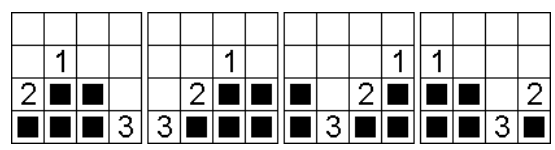

Fig. 6. 128-bit user key recovery

Next we calculate byte 2 of $T_{1}$ using the positions in row 2 where we find a a symbol (the symbols 2 in figure 6). We can check the correctness of byte 0 by 
checking 8 additional symbols in row 2 (the black squares in row 2 in figure 6) since we have the formulae:

$$
\begin{aligned}
\underline{\mathrm{a} \oplus} \underline{1^{\prime} \oplus \mathrm{b}} & =\left(0^{\prime} \oplus 1^{\prime} \oplus \mathrm{a} \oplus \mathrm{b}\right) \oplus \mathrm{a} \oplus\left(0^{\prime} \oplus 1^{\prime} \oplus \mathrm{a} \oplus \mathrm{b}\right) \oplus 1^{\prime} \oplus \mathrm{b} \\
& =\left(0^{\prime} \oplus 1^{\prime} \oplus \mathrm{a} \oplus \mathrm{b}\right) \oplus 0^{\prime} \\
& =\underline{0^{\prime}} \\
\underline{1^{\prime}} \oplus \underline{0^{\prime}} \oplus \mathrm{a} & =\ldots \\
& =\underline{\mathrm{b}} .
\end{aligned}
$$

and $\mathrm{a}$ and $\mathrm{b}$ are known in advance.

Finally we calculate byte 3 of $T_{1}$ using the formula $s_{0} \oplus s_{1} \oplus s_{2}=s_{3}$ with $s_{0} s_{1} s_{2} s_{3}$ a permutation of the symbols $\left\{\underline{0^{\prime}}, \underline{1^{\prime}}, \underline{\mathrm{a}}, \underline{\mathrm{b}}\right\}$. If we obtain four times the same value for byte 3 (in each of the four columns), we have found $T_{1}$. If we obtain several different values for byte 3 of $T_{1}$ the initial assumption of byte 0 of $T_{1}$ was wrong and we have to continue guessing it.

If we have found a correct value for $T_{1}$ then we have found the state $U^{\prime}$ and $U$ completely. So we can calculate $T_{0}=\bigoplus_{i=0}^{3} U[i]$ so we have state $V^{\prime}$ and finally state $V . V[2]$ and $V[3]$ should both be $0 x 00000000$. 